\title{
Space Charge Limited Currents Calculations in Coaxial Cylindrical Diodes Using Particle-in-Cell Simulations
}

\author{
S. Mahalingam*, C. Nieter, J. Loverich, D. Smithe and P. Stoltz
}

Tech-X Corporation, Boulder, Colorado, USA

\begin{abstract}
Three dimensional particle-in-cell (PIC) simulations are conducted to measure the space charge limited (SCL) currents in coaxial cylindrical diodes. New cut-cell emission and absorption boundary algorithms implemented in the electromagnetic PIC code, VORPAL are utilized for this study. Comparisons of VORPAL SCL current results are made with i) theoretical SCL current results for the one dimensional planar diode, ii) analytical solutions of SCL current results for the one dimensional and two dimensional cylindrical diode cases, and iii) an experimental cylindrical diode operated as a virtual cathode oscillator. VORPAL SCL current results show agreement within a few percent with the analytical two dimensional cylindrical diode results for the non-relativistic operating voltages of 1-100 kV and within error bars with the experimental cylindrical diode results for the diode operating voltage of $340 \mathrm{kV}$.
\end{abstract}

\section{INTRODUCTION}

The measurement of space charge limited (SCL) current density for the cylindrical diodes is useful for the development of high power microwave devices, vacuum electronics, vircator and number of other plasma applications. The SCL current has been theorized [1,2] and numerically studied [3-5] well for the 1-D, and 2-D planar diode applications. Similarly theoretical $[6,7]$, analytical [8] and numerical studies $[9,10]$ were performed for measuring the SCL current in coaxial cylindrical diodes. But the coaxial cylindrical diode SCL current studies were either based on the power series assumption made by Langmuir and Blodgett [6] and by Page and Adams [7] for the 1-D cylindrical diode or based on the approximate analytical solutions obtained for the 1-D and 2-D cylindrical diodes [8]. Also in reference [9], 2-D PIC simulations were conducted to measure 2-D effects of SCL currents in coaxial cylinders with a finite emitter width in the low voltage and low current operating regime. Numerical PIC codes such as KARAT [11], OOPIC [12] and number of other tools were used in the past for the SCL current studies in both planar diodes and cylindrical diodes in 1-D and 2-D. In this work, we extend the SCL current measurement in three dimensions using VORPAL [13], which allows 3-D PIC simulations of coaxial cylindrical diodes. These simulations are possible due to new chargeconserving emission and absorption algorithms, and part of the goal of this work is to benchmark these algorithms. Presently other PIC codes [12] require separate divergence correction steps along with the electromagnetic update steps to maintain charge conservation in electromagnetic simulations and represent the curved geometries using stairstepped boundaries that make stair-stepped emission profile. The new cut-cell emission and absorption algorithms in VORPAL eliminate the requirement of separate divergence corrector and correctly represent the curved surfaces so that

*Address correspondence to this author at the Tech-X Corporation, Boulder, Colorado, USA; E-mail: sudhakar@txcorp.com accurate emission profile is obtained in PIC simulations. This SCL current study looks in to the aspects of operating conditions such as the varying gap voltage, the characteristic impedance (i.e., by altering the A-K gap) and the varying emitter width under the non-relativistic regime of the cylindrical diode operation. The numerical SCL current results are compared for the cylindrical diode with the theoretical predictions for the 1-D planar diode, and the analytical predictions for the 1-D and 2-D cylindrical diodes.

In this paper, first we present the basic governing equations established for the SCL current in planar and cylindrical diodes. Then we give the details of the VORPAL, numerical PIC code that is used for the SCL simulations, and discuss new cut-cell emission and absorption algorithms. Next, we discuss the coaxial cylindrical geometry set up that is used in this SCL study and the computational parameters used in the numerical simulations. Also we present the details of experimental coaxial cylindrical diode operated as a virtual cathode oscillator (vircator). Then we provide the VORPAL simulation SCL current results for the coaxial cylindrical diodes and compare the VORPAL SCL current results with theoretical, analytical and experimental SCL current results. VORPAL SCL current results show agreement within a few percent with the analytical two dimensional cylindrical diode results for the non-relativistic operating voltages of $1-100 \mathrm{kV}$ diode with a A-K gap size of $0.005 \mathrm{~m}$ and within error bars with the experimental cylindrical diode results for the diode operating voltage of $340 \mathrm{kV}$.

\section{GOVERNING EQUATIONS}

The 1-D SCL current density in the planar diode, $J_{I D, p 1}$, is given by the Child-Langmuir Law [2]

$$
J_{1 D, p l}=\frac{4}{9} \varepsilon_{0} \sqrt{\frac{2 e}{m}} \frac{V_{a}^{3 / 2}}{d^{2}}
$$

where $\varepsilon_{0}$ is the permittivity of free space, $e$ is the electron charge, $m$ is the electron mass, $V_{a}$ is the A-K gap voltage, and $d$ is the A-K gap distance. Similarly the Langmuir- 
Blodgett's law [6] gives the 1-D SCL current density in the cylindrical diode, $J_{I D, c y l}$,

$$
J_{1 D, c y l}(r)=\frac{4}{9} \varepsilon_{0} \sqrt{\frac{2 e}{m}} \frac{V_{a}^{3 / 2}(r)}{r^{2} \beta^{2}}
$$

where $\beta$ is the geometry correction factor given by the infinite series

$$
\beta=\mu-\frac{2 \mu^{2}}{5}+\frac{11 \mu^{3}}{120}-\frac{47 \mu^{4}}{3300}+\ldots
$$

Here $\mu=\ln \left(r / r_{c}\right)$, and $r_{c}$ is the radius of the cathode cylinder.

In reference [8], Chen et al., provided approximate analytical solutions for the SCL current in 1-D infinitely long cylindrical diodes and 2-D cylindrical diodes.

$$
J_{1 D, C y l}=\frac{4}{9} \varepsilon_{0} \sqrt{\frac{2 e}{m}} \frac{V_{a}^{3 / 2}(r)}{d^{1 / 2} r_{c}^{3 / 2}}\left(\frac{1}{\ln \left(r_{a} / r_{c}\right)}\right)^{3 / 2} \text { for } r_{c}<r_{a},
$$

and

$$
J_{2 D, C y l}=J_{1 D, C y l}\left(1+\frac{2 r_{c}^{2}}{W^{2}}\right) \text { for } W>>r_{c},
$$

Here $r_{a}$ is the radius of the anode cylinder and $W$ is the width of the cathode emission. In reference [8], researchers found that Equations (2) and (3) give identical results. Consequently, we've chosen to use the 1D current density given in Equation (3) for comparisons with VORPAL cylindrical diode simulations. Also Chen et al. [8] noted that the 2-D SCL current density solution given in Equation (4) is valid for large emission widths that satisfy $W>>r_{c}$.

The characteristic impedance [14] of the coaxial cylindrical diode is given by,

$$
Z=\sqrt{\frac{L}{C}}=138.0 \log _{10}\left(r_{a} / r_{c}\right)
$$

where $L$ is the inductance per unit length and $C$ is the capacitance per unit length which are functions of the outer and inner radii of the coaxial cylindrical diode. The unit of characteristic impedance is given in Ohms.

Also in the SCL current studies, the SCL current density are also reported in terms of perveance, $P$, which is the proportionality coefficient between the SCL current, $I$, and the A-K gap voltage $V_{a}^{3 / 2}$. It is given by [15],

$P=\frac{I}{V_{a}^{3 / 2}}=\frac{J A_{s}}{V_{a}^{3 / 2}}$

where $A_{s}$ is the surface area and the unit of perveance is $\mathrm{A}^{-} \mathrm{V}^{-}$ $3 / 2$.

Maxwell's equations for solving the electromagnetic fields are given by [16] $\nabla \cdot E=\frac{\rho}{\varepsilon_{0}}$,

$\nabla \cdot B=0$,

$\frac{\partial B}{\partial t}=-\nabla \times E$,

$\frac{\partial E}{\partial t}=c^{2} \nabla \times B-\frac{j}{\varepsilon_{0}}$.

Here $E$ is the electric field vector, $B$ is the magnetic field vector, $j$ is the current density vector, $c$ is the speed of light, and $t$ is the time. In electromagnetic PIC (EM-PIC) simulations, the magnetic and electric fields are obtained by solving the Faraday's equation (Equation (9)) and AmpereMaxwell's equation (Equation (10)). Equations (7) and (8) need not be solved in the EM-PIC as they are assumed to be satisfied by the continuity equations. In electrostatic PIC (ES-PIC) simulations, the currents are assumed to be small so that no significant self-induced magnetic fields exist and that the charge particle densities do not change quickly relative to the speed of propagation of electromagnetic waves. So in this situation, only Gauss's law (Equation (7)) is solved to get electric fields. In terms of electric potential, $\phi$, the electric field is given as

$E=-\nabla \phi$.

Substituting Equation (11) into Equation (7) gives the Poisson's equation

$\nabla^{2} \phi=-\frac{\rho}{\varepsilon_{0}}$.

The governing equations for the particle motion are given by the classical Newton-Lorentz equations of motion. The charge particle equations of motion in the electric and magnetic fields are [16]:

$m \frac{d u}{d t}=q\left(E+\frac{u \times B}{\gamma}\right)$,

$\frac{d x}{d t}=v$

where $q, u$, and $x$ are the particle charge, particle relativistic velocity vector, and position vector respectively. The relativistic particle velocity is calculated using

$u=\gamma v$

where the relativistic factor, $\gamma$, is $\gamma=1+\frac{v^{2}}{c^{2}}$.

\section{VORPAL PIC CODE}

VORPAL [13] is a 3-D particle-in-cell electromagnetic and electrostatic simulation tool. VORPAL uses finite difference-time domain to solve Maxwell's equations, but includes an advanced technique known as embedded boundaries [17] to allow accurate representation of curved geometries within rectangular grids. VORPAL can solve for 
electromagnetic fields in full $3 \mathrm{D}$, and the embedded boundary methods make VORPAL suited to perform PIC simulations of the SCL current study in coaxial cylindrical diodes. Fig. (1) illustrates the schematic view of the coaxial cylindrical diode simulated using VORPAL. Here the electrons are emitted from the inner cathode cylinder and the outer cylinder is the anode. Electrons are colored based on their relative energies.

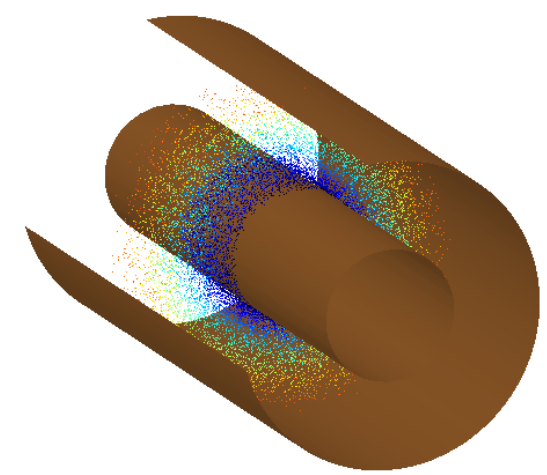

Fig. (1). Schematic view of the coaxial cylindrical diode simulated using VORPAL. These simulations take advantage of new cut cell emission and absorption algorithms, and part of the goal of this work is to benchmark these algorithms.

\subsection{Cut-cell Emission and Absorption Algorithms}

New cut-cell emission and cut-cell absorption algorithms [18] are implemented in VORPAL to handle particle emission and particle kill on embedded boundaries. Conformal boundaries are important for particle emission for a number of reasons. First of all, when field emitters are used it is extremely important to compute the correct electric fields at the boundary to obtain the correct emission current. Also, in many situations it's important to obtain uniform particle emission from curved surfaces, this can be challenging and unnatural using stair stepped boundaries since the emitted particles initially produce a stair-stepped emission profile. Some conformal representation of the boundary is necessary to produce accurate emission profiles. Stair step meshes do have one advantage since it's very easy to design an emission routine that conserves Gauss's law. Particles are simply placed on the edge of the conductor and moved into the solution domain in the stair step boundary case when inverse area weighting is used. For conformal, embedded boundaries it's necessary to move the particles from a region in the conductor where no charge is distributed inside the vacuum region initially.

Fig. (2a-c) show the particle move in a cut-cell geometry. In PIC codes particles contained in a cell have their charge distributed or weighted to computational nodes of the grid for the electromagnetic field update. A very common charge weighting scheme is area weighting whereby a charge is distributed entirely to a single node of the computational grid if the particle lies directly on top of that node, but is otherwise partially distributed to each of the 4 nodes of the computational cell that contains the particle (in 2D) - a description of area weighting can be found in [19]. In these figures red dots indicate non-zero charges distributed from the electrons using area weighting while blue dots represent electrons. Also shaded region represents conductor while the white region represents vacuum. Fig. (2a) shows a particle initialized at a conducting corner. Using area weighting all of the charge is distributed to that same corner. The charge is then moved to the surface of the conductor and then into the vacuum in Fig. (2b). This approach to particle emission preserves Gauss's law in electromagnetic simulations. On the other hand, if a particle is initialized at the cut cell surface charge is distributed to the four corners of the cell including corner inside the vacuum. When the particle is finally moved inside the domain some charge remains at these vacuum corners resulting in unphysical electric field build up so that Gauss's law is no longer preserved. In high emission current simulations the field build up can be large enough to destroy the solution.



Fig. (2). Diagram showing particle move in a cut-cell geometry. Fig. (2a, b) represent a Gauss's law preserving particle move in cutcell geometry during electromagnetic simulations. The particle starts at a conducting corner (a) and is then moved to the surface, then it's moved from the surface into the domain (b). Starting the particle at the cut-cell surface (c) distributes charge inside the domain in the beginning and this charge causes unphysical field buildup at the boundary.

The field inside the conductor is 0 so it's OK to leave charges behind in the conductor since the field equations are not solved there. The move from the conducting corner into the vacuum allows the fields in the first cell to be updated self consistently so that the charge conserving properties of the particle move are maintained inside the vacuum. On the other hand, the charge that is left behind from a move directly from the surface of the cut-cell boundary is not completely eliminated during the charge conserving move, resulting in field build up inside the domain. Absorption is performed in the reverse order. Details of the cut-cell emission and absorption algorithms are given in reference [18].

\section{SIMULATION SET UP}

VORPAL simulations are performed to benchmark these new algorithms by comparing SCL currents in a coaxial cylindrical diode for varying gap voltages $\left(V_{a}\right)$, for varying characteristic coax impedances $(Z)$ and for varying emitter width $(W)$ with the theoretical SCL current of 1-D planar diode and with the analytical solutions of SCL currents of 1$\mathrm{D}$ cylindrical, and 2-D cylindrical diodes, and to benchmark the VORPAL SCL current results with experimental SCL current measurements of a coaxial cylindrical diode.

A cylindrical geometry with the anode radius larger than the cathode radius is chosen. For the varying voltage simulations, the anode radius is $0.01 \mathrm{~m}$ and the cathode radius is $0.005 \mathrm{~m}$ that gives an A-K gap of $0.005 \mathrm{~m}$. The emitter width is $0.02 \mathrm{~m}$ for both varying A-K gap voltage study and the varying characteristic impedance study. The electric potential of the cathode cylinder is maintained at 0 
volts and the electric potential of the anode cylinder is varied to obtain the desired gap voltages. Seven A-K gap voltage cases $0.1 \mathrm{kV}, 0.5 \mathrm{kV}, 1 \mathrm{kV}, 5 \mathrm{kV}, 10 \mathrm{kV}, 50 \mathrm{kV}$, and $100 \mathrm{kV}$ are considered. For both the varying characteristic impedance and varying emitter width SCL current simulations, a constant A-K gap voltage of $1 \mathrm{kV}$ is considered. Five different A-K gap distances $0.001 \mathrm{~m}$, $0.0025 \mathrm{~m}, 0.005 \mathrm{~m}, 0.0075 \mathrm{~m}$, and $0.009 \mathrm{~m}$ are considered by varying the radius of the cathode cylinder which gives the following five coax characteristic impedance $6.3 \mathrm{Ohms}, 17.2$ Ohms, 41.5 Ohms, 83.0 Ohms, 138.0 Ohms cases based on Eq. (5). Seven emitter width cases $0.0025 \mathrm{~m}, 0.005 \mathrm{~m}, 0.01$ $\mathrm{m}, 0.02 \mathrm{~m}, 0.03 \mathrm{~m}, 0.04 \mathrm{~m}$, and $0.05 \mathrm{~m}$ are considered for the emitter width SCL simulations and the cathode radius is kept at $0.005 \mathrm{~m}$ which gives emitter width ratios $\left(\mathrm{W} / \mathrm{r}_{\mathrm{c}}\right)$ of $0.5,1$, 2, 4, 6, 8 and 10 .

A uniform computational grid is chosen in which the number of grid points in the axial direction is 10 , and the number of grid points in both transverse directions are 50. More computational grid points are included in the $\mathrm{y}$ and $\mathrm{z}$ directions to sufficiently resolve the curved surfaces of the coaxial cylinders. This computational grid has a grid resolution of $2.87 \times 10^{-4} \mathrm{~m}$, a time step size of $0.47 \mathrm{ps}$ and a Dey-Mittra fractional time step factor of 0.5 . These computational parameters are selected such that they satisfy the numerical stability limits such as Courant-FriedrichsLewy (CFL) condition. The number of macro particles per computational cell in the simulation is 20 and the macro particles are weighted with $10^{5}-10^{8}$ physical particles. Time integration of charge particle equations of motion given in Equations (13)-(14) is handled using a relativistic Boris advance technique [19]. The cathode electron emission current density is spatially uniform and all electron macro particles are emitted with zero kinetic energy. The electrons are absorbed at the axial boundaries. The electric fields are self-consistently modeled either using an electrostatic algorithm or electromagnetic algorithm, and we compare the results from the two as an additional check on the algorithms. The EM algorithm solves the full set of Maxwell's equations consistently without making any assumptions. It includes the electric fields produced by time varying magnetic fields and the magnetic fields produced by time varying electric fields. It also determines the current values. The ES algorithm models only the dynamic electric fields and does not model the dynamic magnetic fields. The ES algorithm assumes that the currents are small so that no significant self-induced magnetic fields exist. Also, the charge particle densities do not change fast relative to the speed of propagation of electromagnetic waves. So the choice of electric solver algorithm is based on the operating parameters such as the significance of self induced magnetic field effects. When the electromagnetic algorithm is used for the SCL current study, perfectly matched layer (PML) boundary conditions are taken at both ends along the axial direction of coaxial cylinders to control the numerical reflection propagating in to the computational domain. Also in the electromagnetic simulations, current sources are applied to create the desired A-K gap electric field and a feedback system [18] is enabled to control the applied voltage between the A-K gap. In these simulations the voltage is calculated by integrating the electric field across the gap, the voltage is then modified by increasing or decreasing the current applied at the boundaries during the simulation. When particle current exists, the voltage drops so the current source at the boundaries compensates for this drop in voltage and constant voltage is maintained throughout the simulation. Adjustment of the current source is accomplished through a feedback class implemented in VORPAL. In these simulations the following feedback factor calculation is performed

$f^{n+1}=f^{n}+\frac{\Delta t}{\tau} \frac{(D-M)}{(|D|+|M|)} f^{n}$

where $f^{n}$ is the feedback factor at time level $n$. D is the desired voltage across the gap, $M$ is the measured voltage across the gap at time level $n, \tau$ is the relaxation time and $\Delta t$ is the integration time step for the simulation. This feedback factor is then used to scale the current source at the boundaries (not the particle emission current) so that $J^{n}=f^{n} J_{0}$ where $J$ is the scaled current density and $J_{0}$ is the initial current density.

An initial guess is used for the SCL current density values the value from the 1-D Child-Langmuir's equation given in Equation (1). The simulation times are selected to ten times the time required for an electron to cross from the cathode cylinder to the anode cylinder. The current density values are increased until the applied current density exceeds the space charge limit. To know whether an applied current density is exceeding or not exceeding the coaxial cylindrical diode SCL current density, all electron macro particles direction of travel along the radial direction are checked. When the current density exceeds the space charge limit value, the created electrons near the cathode experience the electric field created by the electrons before it and they get decelerated. These decelerated electrons reflect and return to the cathode. This creates a virtual cathode formation near to the cathode surface. This limits the amount of current emission from a surface only emitting electrons. If none of the electrons are found reversing to the cathode then it can be taken that the applied current density is not exceeding the SCL and can be taken as no formation of virtual cathode. If this is the case, then the specified current density is increased and VORPAL simulation is repeated. If more than $1 \%$ of the total electrons are found reversing towards the cathode, then the applied current density value can be taken as that it exceeds the SCL current density of the coaxial cylinders. In this case, the applied current density value is lowered and VORPAL simulation is repeated. This trial and error process is continued until the SCL current value is determined for the coaxial cylinders. It should be realized that this trial and error process could have accuracy error as the final selected SCL current results could be differing within a few percentage $(+$ or $-1-2 \%)$ with the actual SCL currents.

Figs. $(3,4)$ show the particle radial phase-space velocity results along the radial gap between the coaxial cylinders. Each blue dot in Figs. $(\mathbf{3}, \mathbf{4})$ represents a macro electron particle. Fig. (3) represents the case where the applied current density value is below the SCL current of the coaxial cylindrical diode. No virtual cathode formation is seen near the cathode radial location of $5 \mathrm{~mm}$ as none of the electrons decelerated back to the cathode (i.e., negative radial velocity values). Fig. (4) represents the case where the applied current 
density exceeds the SCL current of the coaxial cylindrical diode. It is clearly shown in Fig. (4) that virtual cathode is formed near the cathode radial location of $5 \mathrm{~mm}$ as few of the electrons are found to have negative radial velocity values.



Fig. (3). Electron radial phase-space velocity results in $\mathrm{m} / \mathrm{s}$ across the radial gap between the coaxial cylindrical diode for the case when the applied current density does not exceed the SCL current density. The physical parameters are: $V_{a}=0.1 \mathrm{kV}, d=5 \mathrm{~mm}, r_{a}=10$ $\mathrm{mm}, r_{c}=5 \mathrm{~mm}$, and $j=185 \mathrm{~A} / \mathrm{m}^{2}$.

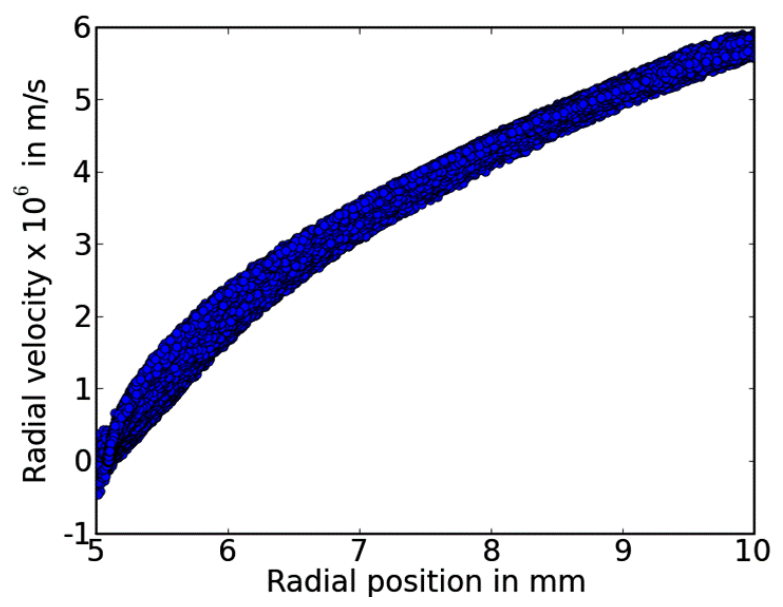

Fig. (4). Electron radial phase-space velocity results in $\mathrm{m} / \mathrm{s}$ across the radial gap between the coaxial cylindrical diode for the case when the applied current density does exceed the SCL current density. The physical parameters are: $V_{a}=0.1 \mathrm{kV}, d=5 \mathrm{~mm}, r_{a}=10$ $\mathrm{mm}, r_{c}=5 \mathrm{~mm}$, and $j=200 \mathrm{~A} / \mathrm{m}^{2}$.

\section{RESULTS AND DISCUSSIONS}

In this section, first SCL current density results for the varying gap voltages and varying characteristic coaxial impedances are presented. The SCL current density results for the coaxial cylindrical diode are compared with the theoretical 1-D planar diode SCL current density, the analytical 1-D cylindrical diode SCL current density and the analytical 2-D cylindrical diode SCL current density results. Then the details of a comparison of simulation with an experimental coaxial cylindrical high power diode operated as a vircator are given.

\subsection{SCL Current Study}

Figs. (5-7) show the SCL current density results from VORPAL PIC simulations of different A-K gap voltages, different coaxial characteristic impedances and different emitter widths respectively. The SCL current density values are increasing with increasing gap voltages and the trend line fitted to the VORPAL results indicates that the current density is proportional to the voltage as $\mathrm{J} \sim \mathrm{V}^{3 / 2}$ which matches well with the SCL current density results given in Equations (1)-(3). Fig. (6) shows that SCL current results are decreasing for increasing values of coaxial characteristic impedances (up to $41.5 \mathrm{Ohms}$ ). But for larger coaxial characteristic impedances, the SCL current density values are found to be increasing. A smaller coaxial impedance means that the gap between the anode and the cathode cylinders is smaller. As the A-K gap becomes smaller compared to the cathode radius, the coaxial cylinder problem looks almost similar to the 1-D parallel plate. The VORPAL results indicate this behavior for the smaller coax impedance case $(Z=6.3$ Ohms, $d=0.001 \mathrm{~m})$ as shown in Fig. (6), where the 1-D theoretical current density for planar diode is agreeing closely. For the last three coax impedance cases where the cathode radii values are made smaller for rising the coax impedances has resulted in the rise of SCL currents. The SCL current density values increase for the smaller emitter width cases (for $\mathrm{W} / \mathrm{r}_{\mathrm{c}}=0.5$ and 1 ) and mostly remain unchanged for all other emitter width cases tested. Chen et al. [8] had noted that the 2-D analytical solution for the cylindrical diode given in Equation (4) is valid for $W>r_{c}$. This condition is observed to be true in Fig. (7) where the 2D analytical solution gives erroneous SCL current results for the smaller emitter width cases. VORPAL simulations disagree with the 1-D cylindrical infinite diode of Eq. 3 by 13-36\%. This discrepancy between VORPAL and the 1-D cylindrical infinite diode solution likely due to the finite length of the simulation domain considered. At low emitter width cases, VORPAL results are agreeing closer to the 1-D cylindrical infinite diode. In this emitter width ratio study, the A-K gap is $0.005 \mathrm{~m}$ same as the cathode radius. Since both the smaller emitter width values and the smaller A-K gap make the VORPAL results closely agreeing to the 1-D cylindrical infinite diode solution for the low emitter width cases. If a larger A-K gap is picked for the emitter width study, VORPAL SCL current results could be differing from the 1-D cylindrical infinite diode SCL solutions.

Fig. (8) shows the ratio of the computed SCL currents and i) the theoretical SCL current for the 1-D planar diode (Eq. 1), ii) an analytical solution of SCL current for the 1-D cylindrical diode, (Eq. 3) and iii) an analytical solution of SCL current for the 2-D cylindrical diode (Eq. 4). For this study, the cathode emitter width is taken as $0.02 \mathrm{~m}$ and the $\mathrm{A}-\mathrm{K}$ gap is taken as $0.005 \mathrm{~m}$. The ratio of the simulated current results and the 1-D planar diode results stay within a few percent of 2.0 for all the A-K gap voltages studied. The $1-\mathrm{D}$ cylindrical infinite diode results are roughly $10-20 \%$ below the simulated results over the voltage range considered. This discrepancy is likely due to the finite length of the simulation domain, as the ratio of VORPAL result and 




Fig. (5). VORPAL SCL current density results for the varying A-K gap voltages of coaxial cylindrical diode. Both axes are given in logarthmic scale. VORPAL results indicate that the SCL current density values are increasing with increasing gap voltages (as $\mathrm{J} \sim$ $\mathrm{V}^{3 / 2}$ ).

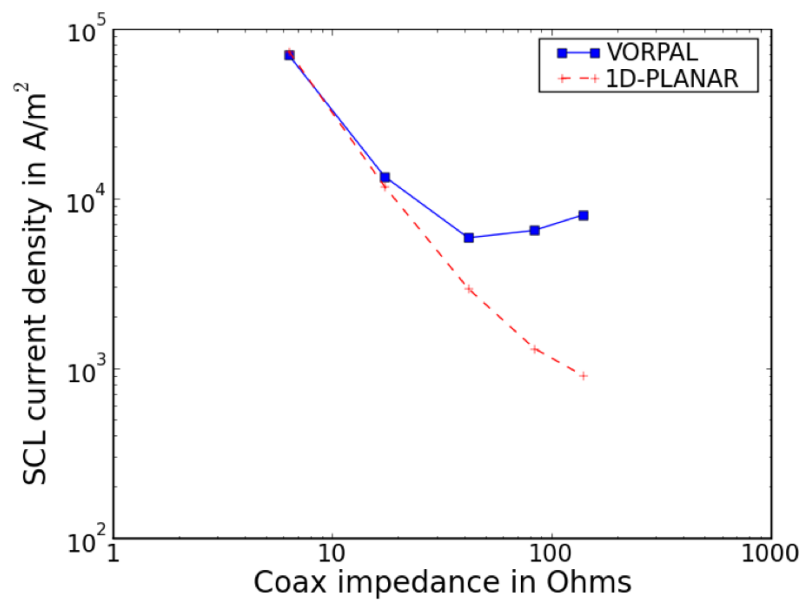

Fig. (6). VORPAL SCL current density results for the varying coaxial characteristic impedances of coaxial cylindrical diode. The A-K gap voltage used for this study is $1 \mathrm{kV}$. As the A-K gap becomes smaller compared to the cathode radius (i.e., smaller coax impedance values), the coaxial cylinder problem looks almost similar to the 1-D parallel plate. The VORPAL results indicate this behavior for $\mathrm{Z}=6.3 \mathrm{Ohms}$ case $\left(\mathrm{d}=0.001 \mathrm{~m}, \mathrm{r}_{\mathrm{c}}=0.009 \mathrm{~m}\right)$ where the 1-D theoretical planar diode of Eq. 1 agrees within 5\%.

the analytical solution of 2-D cylindrical diode are between $0.97-1.05$. The dips observed in Fig. (8) on all SCL current ratio curves for the regions between $0.5-1 \mathrm{kV}$ and $50-100 \mathrm{kV}$ could be due to the accuracy error of the trial and error process used for the SCL current determination.

\subsection{Experimental Cylindrical Diode SCL Current Study}

As a final benchmark of the simulation results, we compare experimental results from a coaxial cylindrical diode [20]. In reference [20], the authors present experimental studies on a high-power coaxial cylindrical diode used for producing intense relativistic electron flow in a vircator regime. They present perveance data for the diode

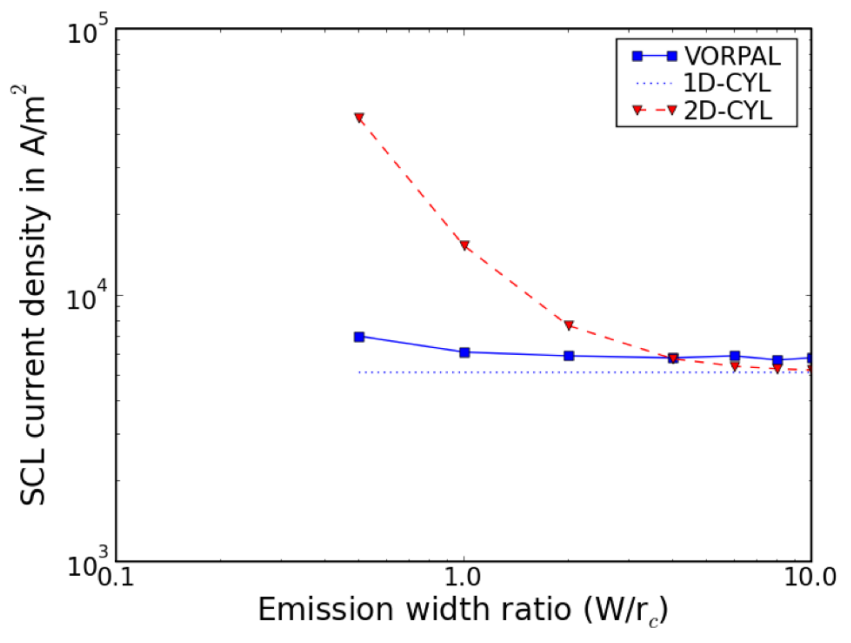

Fig. (7). VORPAL SCL current density results for the varying emitter widths of coaxial cylindrical diode. The A-K gap voltage used for this study is $1 \mathrm{kV}$. VORPAL simulations agree within 1$10 \%$ with the $2 \mathrm{D}$ cylindrical theory of Eq. 4 for $\mathrm{W} / \mathrm{r}_{\mathrm{c}}>4.0$. We find that for $\mathrm{W} / \mathrm{r}_{\mathrm{c}}<2.0$, VORPAL simulation results and Eq. 4 begin to disagree by factors of 2 or more. VORPAL simulations disagree with the 1-D cylindrical infinite diode of Eq. 3 by $13-36 \%$.

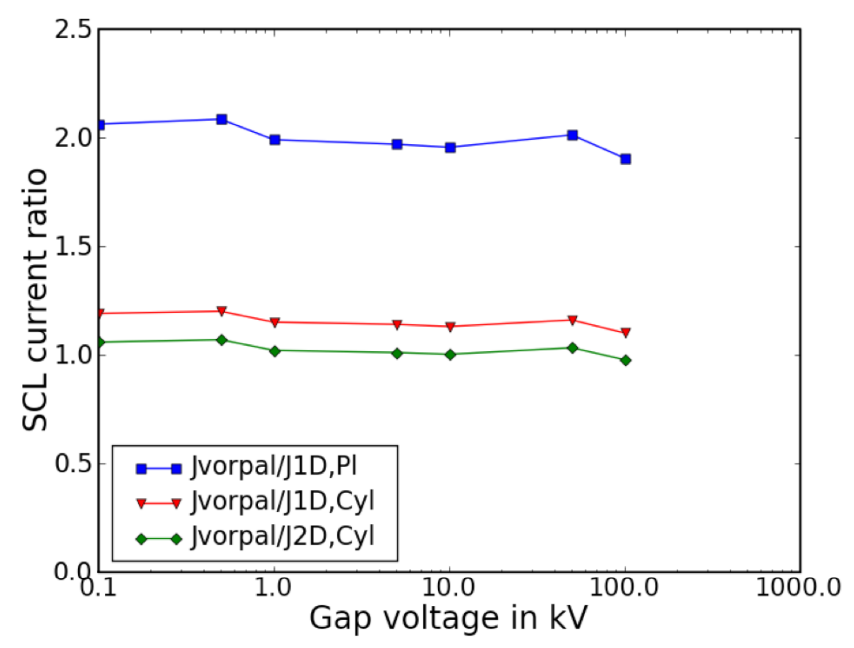

Fig. (8). SCL current ratio results for varying A-K gap voltages. VORPAL simulations significantly differ from the 1-D planar diode theory of Eq. 1 by more than $100 \%$ and differ from the $1-\mathrm{D}$ cylindrical infinite diode theory of Eq. 3 by $10-20 \%$. However they closely agree within $5 \%$ with the 2-D cylindrical theory of Eq. 4 that is likely due to the finite length of the diodes considered in simulations.

operating under different input voltages and different anodecathode $(\mathrm{A}-\mathrm{K})$ gap values. We simulated this experimental cylindrical diode set up with a geometry of inner diameter of $0.123 \mathrm{~m}\left(r_{c}=0.0615 \mathrm{~m}\right)$ and the copper anode mesh cylinder has a diameter of $0.086 \mathrm{~m}\left(r_{a}=0.043 \mathrm{~m}\right)$. This gives an A-K gap of $0.0185 \mathrm{~m}$. The simulated emission width $(W)$ on the cathode cylinder is $0.02 \mathrm{~m}$, and the diode voltage is $340 \mathrm{kV}$.

A uniform computational grid that has 20 grid points in the $\mathrm{x}$-direction and 50 grid points in both $\mathrm{y}$ and $\mathrm{z}$ directions is used. This gives a 3-D grid spacing value of $1.33 \mathrm{~mm}$. The electric fields are self-consistently modeled using the electrostatic algorithm. The time step size is $0.88 \mathrm{ps}$, which 
ensures that the electrons do not cross more than one computational cell in a given time step. All other simulation parameters are same and a similar procedure is followed to determine the SCL current density as applied in the previous SCL current simulations.

For these parameters, the SCL current density is found to be $1.2 \times 10^{6} \mathrm{~A} / \mathrm{m}^{2}$. This gives a perveance result of $4.68 \times 10^{-5}$ $\mathrm{A} / \mathrm{V}^{3 / 2}$. The experimental perveance value from the figure given in reference [20] is between $3.5 \times 10^{-5}-5.0 \times 10^{-5} \mathrm{~A} / \mathrm{V}^{3 / 2}$. Thus the VORPAL numerical result is within the experimentally measured range.

\section{CONCLUSIONS}

In this work 3-D PIC simulations using the VORPAL code are conducted to study the SCL current in coaxial cylindrical diodes, using new charge-conserving emission and absorption algorithms for embedded boundaries. The results agree within a few percent with the analytical solutions of SCL current results given for the 2-D cylindrical diodes for the non-relativistic diode operating voltages of $0.1-100 \mathrm{kV}$ and A-K gap size of $0.005 \mathrm{~m}$. Finally, we show that results are within experiment error bars when comparing with published perveance measurements for a high-power cylindrical diode operating at diode voltage of $340 \mathrm{kV}$.

\section{ACKNOWLEDGEMENTS}

The authors would like to thank Tech-X Corporation for the financial support of this project. Also the authors would like to thank the VORPAL Team.

\section{REFERENCES}

[1] Child CD. Discharge from hot CaO. Phys Rev 1911; 32: 492.

[2] Langmuir I. The effect of space charge and initial velocities on the potential distribution thermionic current between parallel plane electrodes. Phys Rev 1923; 21: 419.
[3] Watrous JI, Luginsland JW, Sasser III GE. Current and current density of a finite-width, space-charge-limited electron beam in two-dimensional, parallel-plate geometry. Phys Plasmas 2001; 8: 289.

[4] Umstattd RJ, Luginsland JW. Two-dimensional space-chargelimited emission: beam-edge characteristics and applications. Phys Rev Lett 2001; 87: 145002.

[5] Lau YY. Simple theory for the two-dimensional Child-Langmuir law. Phys Rev Lett 2001; 87: 278301.

[6] Langmuir I, Blodgett K. Currents limited by space charge between coaxial cylinders. Phys Rev 1923; 22: 347.

[7] Page L, Adams NI. Space Charge between coaxial cylinders. Phys Rev 1945; 68: 126.

[8] Chen X, Dickens J, Hatfield LL, Choi E, Kristiansen M. Approximate analytical solutions for the space-charge-limited current in one-dimensional and two-dimensional cylindrical diodes. Phys Plasmas 2004; 11: 3278.

[9] Kostov KG, Barroso JJ. Space-charge-limited current in cylindrical diodes with finite-length emitter. Phys Plasmas 2002; 9: 1039.

[10] Wheeler CB. The approach to space charge limited current flow between coaxial cylinders. J Phys A Math Gen 1975; 8: 555.

[11] Tarakanov VP. User's Manual for Code KARAT. Springfield, VA: Berkeley Research Associates 1992.

[12] Verboncoeur JP, Langdon AB, Gladd NT. An object-oriented electromagnetic PIC code. Comp Phys Commun 1995; 87: 199.

[13] Nieter C, Cary JR. VORPAL: a versatile plasma simulation code. J Comp Phys 2004; 196: 448-72.

[14] Elmore WC, Heald MA. Physics of Waves. New York: Courier Dover Publications 1969.

[15] Chao AW, Tigner M. Handbook of Accelerator Physics and Engineering. World Scientific 1999; p. 100.

[16] Griffiths DJ. Introduction to Electrodynamics, $2^{\text {nd }}$ ed. Englewood, NJ: Prentice Hall 1989.

[17] Dey S, Mittra R. A locally conformal finite-difference timedomain (FDTD) algorithm modeling three-dimensional perfectly conducting objects. IEEE Microw Guid Wave Lett 1997; 7: 273.

[18] Loverich J, Nieter C, Mahalingam S, Smithe D, Stoltz P. Charge conserving emission from conformal boundaries in electromagnetic PIC simulations. Comp Phys Commun 2009; (to be submitted).

[19] Birdsall CK, Langdon AB. Plasma physics via computer simulation. $1^{\text {st }}$ ed. Bristol: Adam Hilger 1991.

[20] Roy A, Menon R, Mitra S, et al. Intense relativistic electron beam generation and prepulse effect in high power cylindrical diode. J Appl Phys 2008; 103: 014905. 